\title{
Editorial: Advanced Sensing, Materials and Intelligent Algorithms for Multi-Domain Structural Health Monitoring
}

\author{
Chun-Xu Qu ${ }^{1}$, Liang Ren ${ }^{1 *}$, Qingzhao Kong ${ }^{2}$, Yang Zhang ${ }^{3}$, Yunlai Zhou ${ }^{4}$ and \\ Gangbing Song ${ }^{5}$
}

${ }^{1}$ School of Civil Engineering, Dalian University of Technology, Dalian, China, ${ }^{2}$ College of Civil Engineering, Tongji University, Shanghai, China, ${ }^{3}$ School of Physics, Dalian University of Technology, Dalian, China, ${ }^{4}$ Faculty of Enigneering, Universidade Lusófona, Lisbon, Portugal, ${ }^{5}$ Department of Mechanical Engineering, University of Houston, Houston, TX, United States

Keywords: structure health monitoring, sensing, smart materials, intelligent algorithms, engineering

Editorial on the Research Topic

Advance Sensing, Materials and Intelligent Algorithms for Multi-Domain Structural Health Monitoring

Structural health monitoring (SHM) is an effective method to obtain and trace asystem's operation state by sensing its responses during its lifecycle. For decades, SHM has been widely applied to many types of systems, such as bridges, buildings, transmission towers, wharves, machines, and equipment. Furthermore, it is helpful to evaluate structural safety, analyze structural collapse or failure, optimize structural design, and implement structural control. This special issue shares the original research and review articles on the topics of novel point/distributed sensing technology for various engineering fields, advanced nanomaterial and smart functional material and applications in SHM systems, intelligent algorithms, Artificial Intelligence algorithms for data compression, mining and fusing technology, mathematical theories and methods for SHM system, and other related aspects.

SHM attracts much research and has many applications in civil engineering. Jiang et al. propose an active sensing technology based on piezoelectric ceramics for monitoring the bond-slip in old-new concrete interfaces in anchored reinforced concrete structures. Liu et al. analyze the long-term durability monitoring data of high-piled wharves with anode-ladder sensors embedded in the concrete and found that the temperature affects the resistance of concrete significantly. Xiong et al. propose a timber beam crack detection method based on the combination of stress wave sensing and computer vision technology, which can effectively identify the crack severity. Jiang et al. used an optic frequency domain reflectometer technology to measure the distributed strain and used the plane curve reconstruction algorithm to calculate the shape of the deformed pipeline, which can realize the detection of pipeline frost heave deformation. Liu et al. study and compared the failure evolution characteristics of intact coal and fractured coal based on the maximum amplitude distribution of acoustic emission. The research of Wang et al., has shown that a piezoresistive silicon pressure transducer would be suitable for monitoring pore pressure and earth pressure on pile surface during jacking.

SHM can be also used to monitor or detect instruments or equipment components. Liang et al. applied a low-cost miniature impedance board to monitor the threaded pipe connection looseness monitoring and verified its practical performance in impedance measurement and structural damage identification. Chen et al. propose a quantitative monitoring method of bolt looseness based on multi-channel piezoelectric active sensing and an improved convolution neural network. Yin et al. 
employed OFDR technology to locate fatigue hot spots by measuring the maximum curvature of dynamic umbilicus in fatigue tests. Liu et al. propose a real-time structural health monitoring method of a pantograph-catenary system based on strain response inversion, which can accurately calculate the magnitude and location of dynamic contact force between catenary and pantograph. Zhang et al. presente a fiber Bragg grating strain sensor with low temperature sensitivity, whose stain transferring characteristics were conducted by experiment calibration and reliability tests, and the advantages inprecision, reliability, and applicability were verified by on-site tests.

The purpose of SHM is to monitor the structural operation state. To evaluate structural safety and optimize structural design, Cheng et al. present an improved adaptive technology termed the distant relative genetic algorithm for the structural reliability optimization design. Li et al. carried out finite element analysis on the collapse of a transmission tower-line system caused by ice shedding, and systematically studied collapse caused by ice shedding and its influencing parameters. Zhang et al. propose the holistic design scheme of an energy-pile bridge de-icing system based on ontology multi-objective decision making and combined it into an overall design tool. Zhang et al. studied the effect of aftershocks on the fragility of singlestory masonry structures using a probabilistic seismic demand analysis model. Xu et al. propose a fatigue reliability calculation method based on the sequential law and the whole-range S-N curve, where the fatigue reliability of rib-to-deck welded joints of an orthotropic steel deck was evaluated with consideration of the effect of load sequence. Wang et al. applied the novel gradient concrete to the tower of the Chizhou Yangtze River Bridge. The finite element analysis results show that the FGC tower has good mechanical properties and durability for the cable-stayed bridge towers. Wang et al. studied the effects of different diameters on pile tip resistance, pile side resistance, pile axial force, and pile force transmission during static pressure pile penetration.

To make structure safes, structural control is another effective method. Chen et al. have designed a novel damping-limit device to strengthen the purlin roof structure and improve its seismic performance. Gao et al. carried out tensile and compressive tests on a U-shaped steel damper considering different test parameters and derived theoretical calculation formula for its mechanical properties. Sun et al. performed parameter optimization design of a negative stiffness device for highway bridges based on a performance genetic algorithm, which obtained a better shock absorption effect. Ye et al. studied the vibration control performance of viscoelastic materials pounding a tuned mass damper at different temperatures, whose temperature robustness was validated by the experiments.

\section{AUTHOR CONTRIBUTIONS}

All authors listed have made a substantial, direct, and intellectual contribution to the work and approved it for publication.

\section{ACKNOWLEDGMENTS}

All authors' contributions, and those of the editorial staff of Frontiers, are appreciated to make this special issue possible.

Conflict of Interest: The authors declare that the research was conducted in the absence of any commercial or financial relationships that could be construed as a potential conflict of interest.

Publisher's Note: All claims expressed in this article are solely those of the authors and do not necessarily represent those of their affiliated organizations, or those of the publisher, the editors and the reviewers. Any product that may be evaluated in this article, or claim that may be made by its manufacturer, is not guaranteed or endorsed by the publisher.

Copyright (C) 2021 Qu, Ren, Kong, Zhang, Zhou and Song. This is an open-access article distributed under the terms of the Creative Commons Attribution License (CC $B Y)$. The use, distribution or reproduction in other forums is permitted, provided the original author(s) and the copyright owner(s) are credited and that the original publication in this journal is cited, in accordance with accepted academic practice. No use, distribution or reproduction is permitted which does not comply with these terms. 